\title{
Unión cráneo-cervical - anatomía normal y correlación con imágenes
}

\section{Cranio Cervical Junction - Anatomy and Imaging Correlation}

\author{
Nicolás Sgarbi ${ }^{1}$ Osmar Telis ${ }^{1}$ \\ ${ }^{1}$ Departamento Clínico de Radiología, Hospital de Clínicas, \\ Montevideo, Uruguay \\ Address for correspondence Prof. Agregado. Dr. Nicolás Sgarbi, MD, \\ Departamento Clínico de Radiología, Hospital de Clínicas, \\ Montevideo 11600, Uruguay (e-mail: nsgarbi@gmail.com).
}

Rev Argent Radiol 2018;82:161-167.

\section{Resumen \\ Palabras Clave \\ - unión cráneo-cervical \\ - anatomía \\ - trauma cervical \\ - resonancia magnética}

La solicitud de estudios de imagen en pacientes con trauma cervical es muy frecuente en la práctica diaria. Esa patología es causa relativamente frecuente de discapacidad en pacientes jóvenes junto con el trauma encéfalo craneano. En un porcentaje no despreciable de los casos, las lesiones traumáticas comprometen la unión cráneocervical y en esos pacientes, la morbi-mortalidad es más significativa. La transición entre el cráneo y el raquis se basa en un conjunto de estructuras óseas relacionadas por articulaciones muy móviles y estabilizadas por un grupo de ligamentos y músculos que le brindan al mismo tiempo gran solidez. Para una correcta interpretación de los estudios de imagen de uso corriente en la clínica, es fundamental un sólido conocimiento anatómico de la unión cráneo-cervical y sus componentes. Es el objetivo de esta revisión, sistematizar la anatomía de la unión cráneo-cervical con especial énfasis en sus ligamentos, analizar la fisiología de sus movimientos y el concepto de estabilidad para luego realizar una correlación con tomografía computada multi-detector y resonancia magnética.

The request of imaging techniques in patients with cervical spine trauma is very common in clinical practice. Cervical trauma is a relatively common cause of disability in young patients. In a significant percentage of cases traumatic injuries compromise the cranio-cervical junction with more important morbidity and mortality in this group of patients. The transition between the skull and the spine is based on a set of bony structures, high mobility joints, and stabilization mechanism formed by a group of ligaments and muscles. A solid anatomical knowledge of the cranio-cervical junction and its components is essential for a correct interpretation of current high resolution imaging studies. The goal of this review is highlight the anatomy of the cranio-cervical junction with special emphasis on the ligaments, analyze the biomechanics of their movements and the concept of stability. At last but not leastwe will establish a correlation with multidetector computed tomography and high-resolutionmagnetic resonance imaging. received

November 30, 2017

accepted

April 22, 2018

published online

July 19, 2018
DOI https://doi.org/

10.1055/s-0038-1656541.

ISSN 1852-9992.
Copyright $\odot$ 2019, Sociedad Argentina de Radiología. Publicado por Thieme Revinter Publicações Ltda., Rio de Janeiro, Brazil. Todos los derechos reservados.
License terms

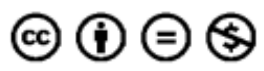




\section{Introducción}

La unión cráneo-cervical (UCC), es el complejo articular que vincula anatómica y funcionalmente al cráneo con el raquis. Es una región única, con una anatomía compleja, que permite combinar solidez y extrema movilidad al mismo tiempo. ${ }^{1,2}$ Está expuesta a múltiples lesiones en casos de trauma y por sus relaciones anatómicas, neurales y vasculares, puede comprometer la vida del paciente. Su estabilidad depende de un delicado conjunto de articulaciones sinoviales y estructuras ligamentarias de disposición compleja. ${ }^{3}$

Es fundamental el detallado conocimiento anatómico de la UCC y sus componentes, sobre todo ligamentarios, para poder realizar una adecuada interpretación de las imágenes y por ello, un preciso diagnóstico. El objetivo de esta revisión es repasar la anatomía normal de la UCC, destacando los componentes más importantes para la estabilidad, sistematizar la fisiología de las articulaciones y sus movimientos y finalmente establecer una correlación con los estudios de tomografía computada (TC) y resonancia magnética (RM) que habitualmente son realizados en el paciente con trauma.

\section{Anatomía Normal}

La UCC se define como el conjunto de estructuras óseas, ligamentarias y musculares que le dan solidez y movimiento a la transición entre el cráneo y la columna cervical. Al mismo tiempo está relacionada directamente con el neuroeje, uno de sus sectores más importantes como es la transición bulbomedular, así como también con ambas arterias vertebrales que son los vasos de origen del sistema vértebro-basilar.

\section{Estructuras Óseas}

La UCC o charnela cráneo-raquídea está formada por los cóndilos del hueso occipital (CO), la $1^{\text {a }}$ vértebra cervical o atlas (C1) y la $2^{\text {a }}$ vértebra cervical o axis (C2). Por eso recibe el nombre de complejo occipito-atloido-axoideo (COAO). Esos 3 huesos están unidos por 5 complejos articulares: ambas articulaciones occipito-atloideas (AOA), la articulación atloido-odontoidea (AAO) y las articulaciones atloidoaxoideas (AAA) (-Fig. 1). Por el lado de la base del cráneo es el hueso occipital el que contribuye a la UCC sobre todo por el foramen magno y los CO que se sitúan a sus lados.

Luego encontramos a C1 formada por un arco anterior y otro posterior con sus masas laterales donde se ubican las superficies articulares que articulan con los $\mathrm{CO}$ y con C2 (-Fig. 2). Por último se suma C2 que contribuye con superficies articulares ubicadas en la cara superior de sus sectores laterales y en la apófisis odontoidea (AO) (-Fig. 3).

\section{Unidad Occipito-atloidea}

Está formada por ambas AOA de disposición lateral, y sus medios de unión. Vincula la base del cráneo con el raquis cervical y está formada por articulaciones de tipo condíleo que se disponen a ambos lados de la línea media de forma simétrica ( - Fig. 4). Por su geometría permite movimientos en los 3 planos del espacio aunque los más amplios son los de flexo-extensión.

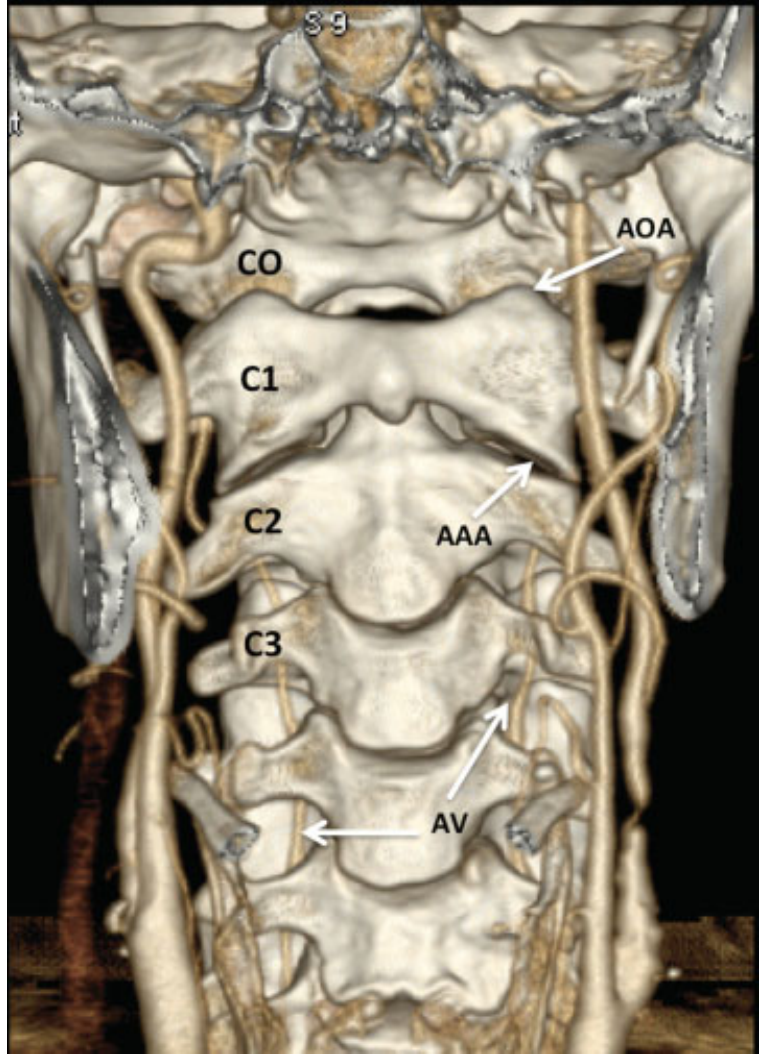

Fig. 1 Tomografía computada multi-detector (TCMD), VR, vista anterior. En el estudio se observa una visión global del sector superior del raquis cervical, vista anterior, con especial interés en la UCC. Se destacan las estructuras que la componen y sus articulaciones, así como las relaciones vasculares. Abreviaciones: AAA, articulación atloido-axoidea; $\mathrm{AOA}$, articulación occipito-atolidea; $\mathrm{AV}$, arteria vertebral; $\mathrm{CO}$, cóndilo occipital; $\mathrm{C} 1,1^{\text {a }}$ vértebra cervical; $\mathrm{C2}, 2^{\text {a }}$ vértebra cervical; C3, 3a vértebra cervical.

\section{Unidad Atloido-axoidea}

Está formada por la AAO y ambas AAA, o sea un total de 3 articulaciones integradas funcionalmente. La AAO se produce entre la $\mathrm{AO}$ de $\mathrm{C} 2$ y una carilla articular pequeña situada en la cara posterior del arco anterior de C1. El compartimiento

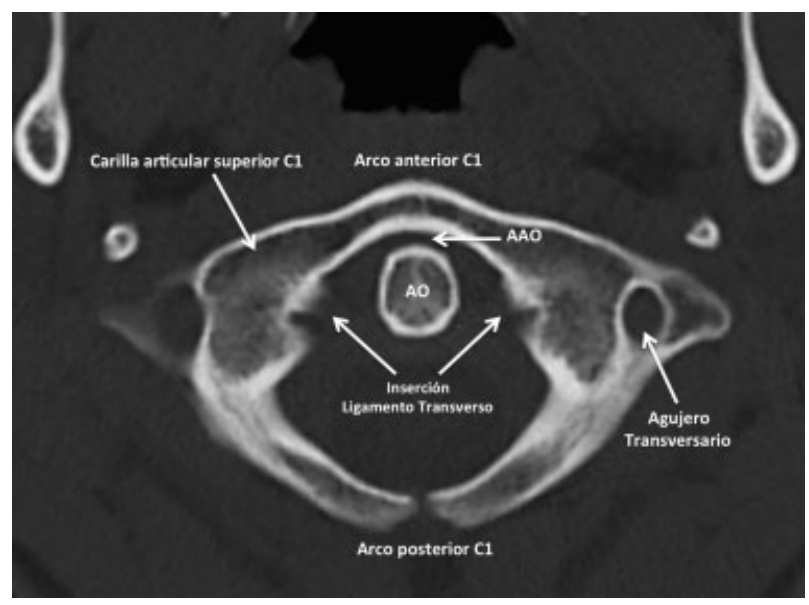

Fig. 2 TCMD, corte axial, ventana ósea, C1. Vista axial de $\mathrm{C} 1$ con sus distintos sectores anatómicos y relaciones principales. Abreviaciones: AAO, articulación atloido-odontoidea; AO, apófisis odontoidea de C2. 


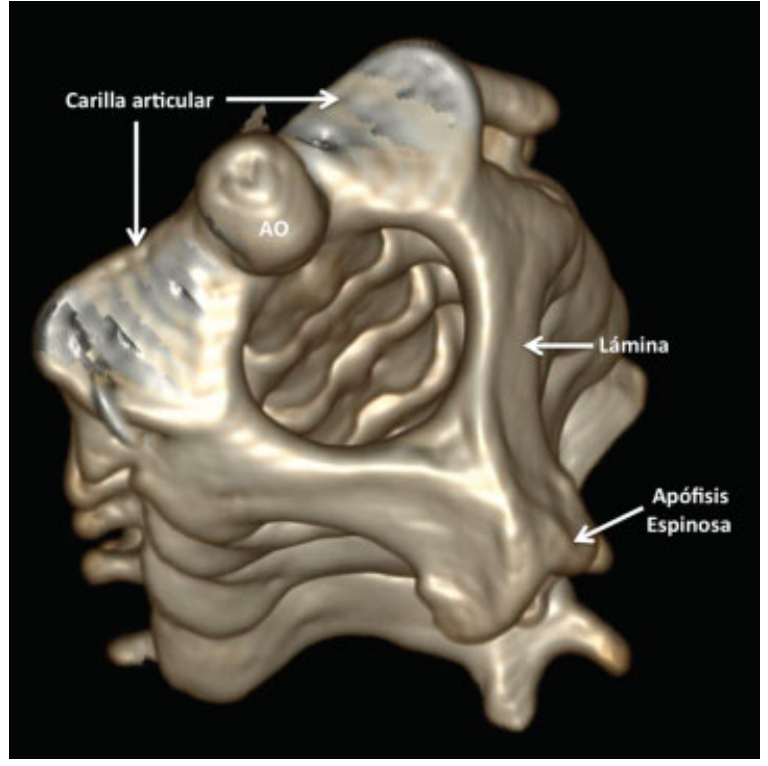

Fig. 3 TCMD, VR, vista oblicua superior de C2. Se muestra la 2a vértebra cervical o axis en una vista desde arriba que muestran sus diferentes sectores de relación con C1. Abreviaciones: AO, apófisis odontoidea de C2.

articular se completa por detrás por el ligamento transverso (LT), que luego revisaremos ( - Fig. 5). Por su geometría, permite sobre todo movimientos de rotación, y la AO por su anatomía y función es el verdadero pívot del raquis sub-occipital. Las AAA, de localización lateral, son equivalentes a las articulaciones inter-articulares del resto del raquis (o facetarias) y son fundamentales ya que acompañan los movimientos de flexoextensión y rotación que se producen en el resto de las articulaciones (-Fig. 6).

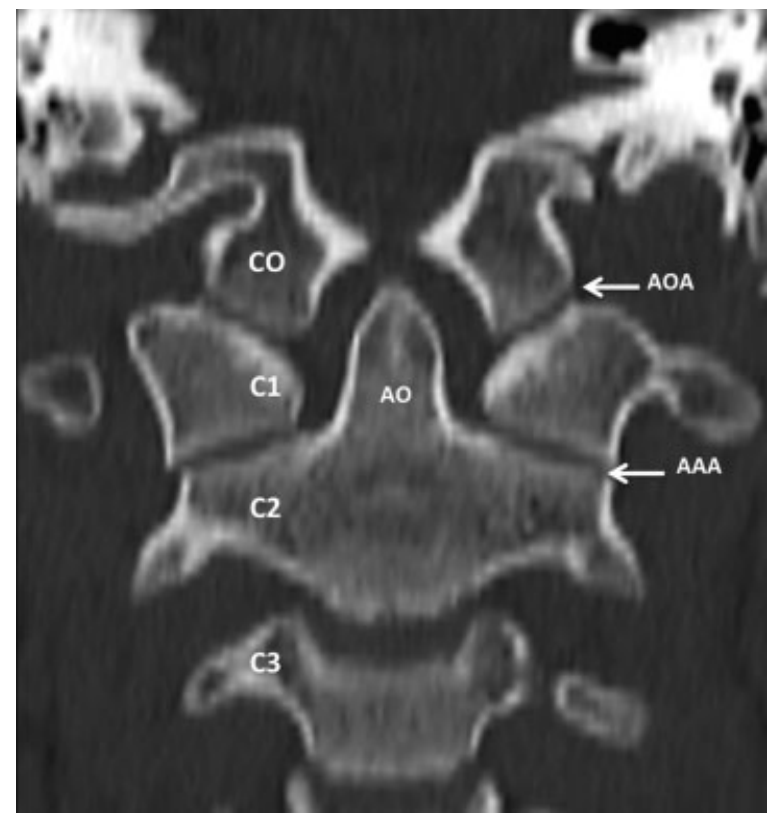

Fig. 4 TCMD, reconstrucción coronal, ventana ósea. Se muestra una vista anterior del raquis sub-occipital y las articulaciones que forman parte de la UCC. Abreviaciones: AAA, articulación atloido-axoidea; AOA, articulación occipito-atolidea; $\mathrm{CO}$, cóndilo occipital; $\mathrm{C} 1,1^{\text {a }}$ vértebra cervical; C2, 2a vértebra cervical; C3, 3a vértebra cervical.

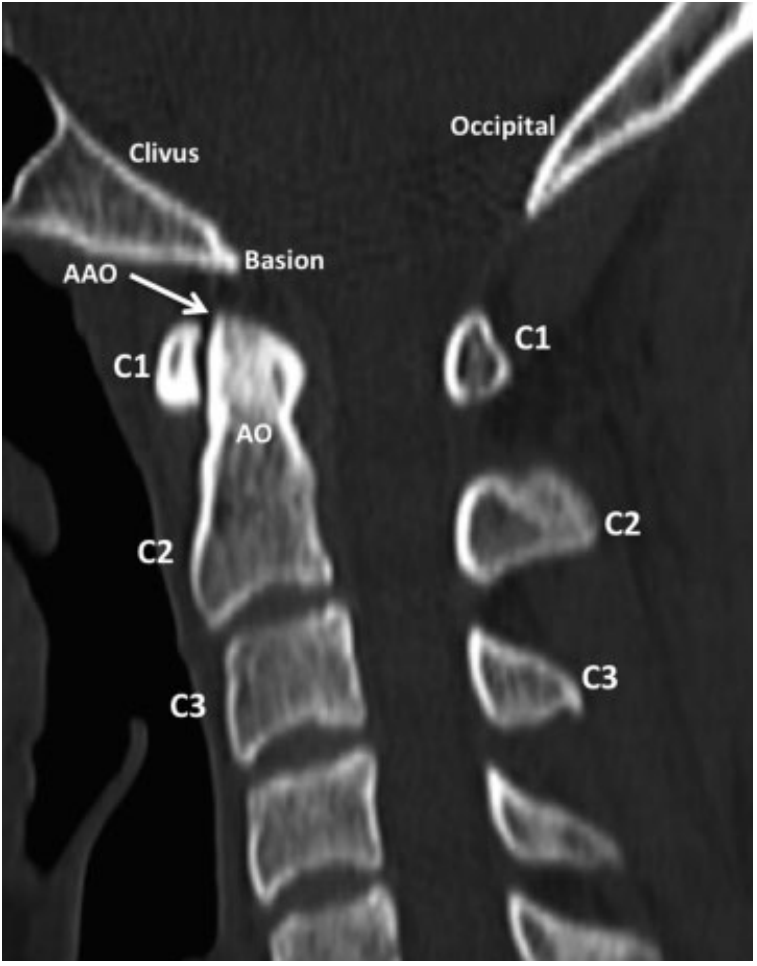

Fig. 5 TCMD, reconstrucción sagital, ventana ósea. En la vista lateral se visualiza correctamente otra articulación de la UCC, una de las más importantes, la $\mathrm{AAO}$, entre el arco anterior de $\mathrm{C} 1$ y la $\mathrm{AO}$ de $\mathrm{C} 2$. Abreviaciones: $\mathrm{AAO}$, articulación atlanto-odontoidea; $\mathrm{AO}$, apófisis odontoidea; $\mathrm{C} 1,1^{\text {a }}$ vértebra cervical; $\mathrm{C2}, 2^{\text {a }}$ vértebra cervical; $\mathrm{C}$, 3a vértebra cervical.

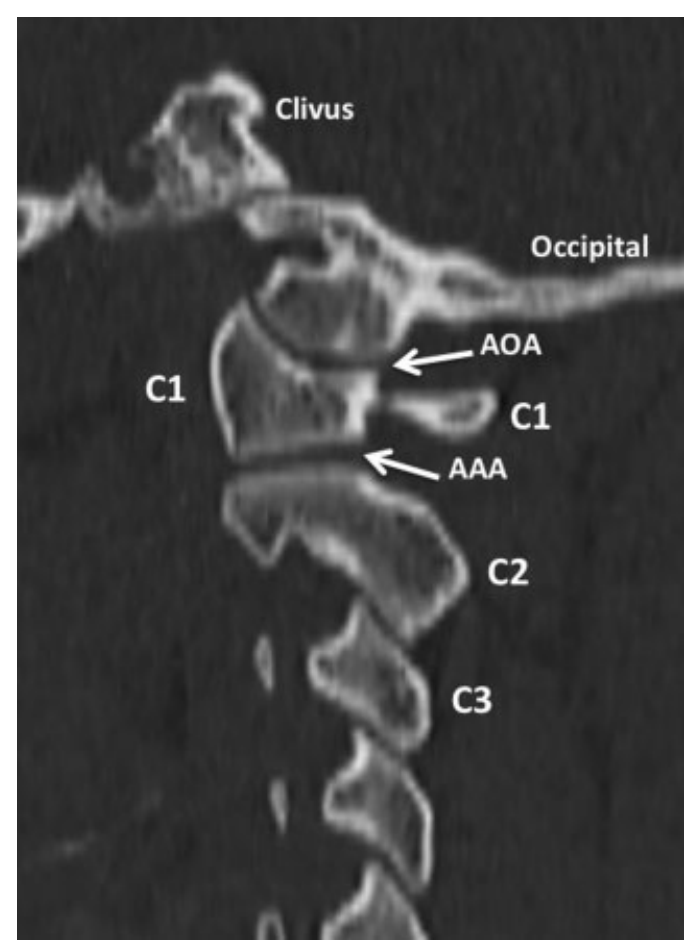

Fig. 6 TCMD, reconstrucción sagital, ventana ósea. En este corte, lateral a la línea media, se identifican las articulaciones que forman parte de la UCC: AOA y AAA. Abreviaciones: $\mathrm{C} 1,1^{\text {a }}$ vértebra cervical;

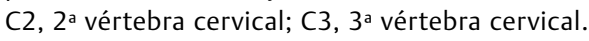




\section{Ligamentos}

Los ligamentos relacionados con la UCC son fundamentales para su estabilidad y los más importantes son el ligamento cruciforme (LC) y los ligamentos alares (LA). En segundo término aportan estabilidad el ligamento longitudinal posterior (LLP) y la membrana tectoria (MT) ${ }^{4,5}$ Existen otro grupo de estructuras secundarias como el ligamento longitudinal anterior (LLA) y sus extensiones craneales, asî como también el ligamento apical (LAp) y la membrana occipito-atloidea posterior (MOAP).

Algunos autores señalan la existencia de un grupo de ligamentos accesorios, de menor desarrollo, que si bien son identificables durante disecciones anatómicas, no son visualizados en estudios de RM de alta resolución. Destacaremos los ligamentos más importantes en la fisiología de la región e identificables en los estudios de RM (-Figs. 7a-10).

Ligamento cruciforme: está compuesto por fibras de disposición transversal y otras verticales lo que le da su nombre (cruciforme o en cruz). Para algunos autores es considerado como el principal estabilizador del raquis suboccipital.

Su componente horizontal es el denominado ligamento transverso (LT) que junto con el arco anterior de C1 forma el compartimiento osteo-fibroso donde se ubica la AO. ${ }^{6}$ Es el ligamento más desarrollado de la UCC y el actor más importante en la estabilidad de la región. Se inserta en los tubérculos laterales de $\mathrm{C} 1$ pasando por detrás de la $\mathrm{AO}$ con un

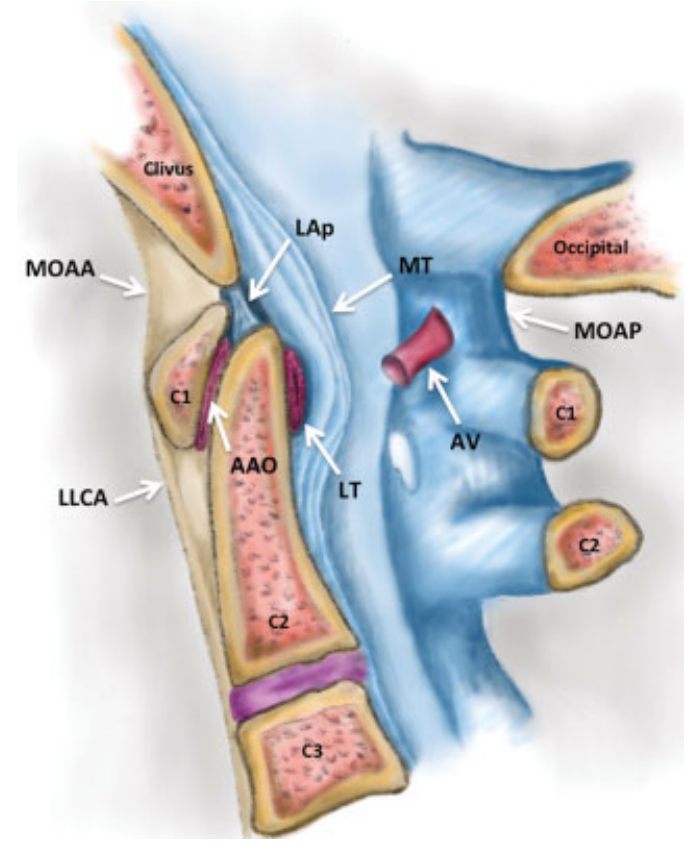

Fig. 7 Esquema sagital de los ligamentos de la UCC. En el esquema se representa un corte sagital a nivel de la línea media donde se muestra la disposición de los principales ligamentos de la UCC, según se describe en el texto. Abreviaciones: AAO, articulación atloidoodontoidea; $\mathrm{AV}$, arteria vertebral; $\mathrm{C} 1,1^{\text {a }}$ vértebra cervical; $C 2,2^{\mathrm{a}}$ vértebra cervical; C3, 3a vértebra cervical; LAp, ligamento apical; LLCA, ligamento longitudinal común anterior; LT, ligamento trasverso; MOAA, membrana occipito-atloido-axoidea; MOAP, membrana occipito-atloidea posterior; MT, membrana tectoria.

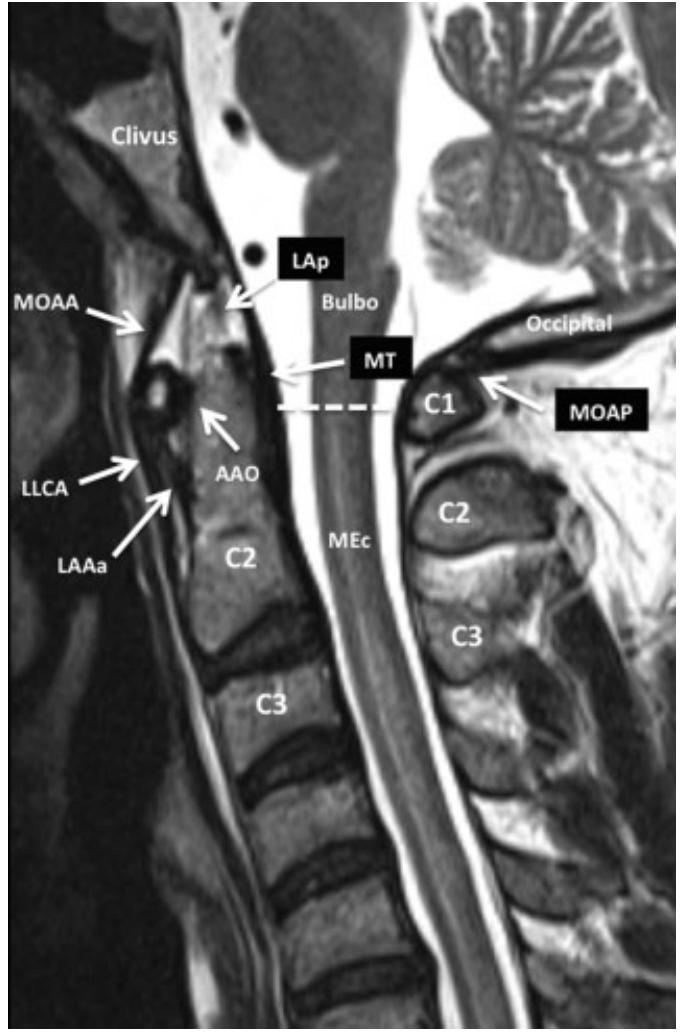

Fig. 8 Resonancia magnética (RM), secuencia ponderada en FSE T2, plano sagital. Corte sagital de la UCC en línea media para visualizar las estructuras de la UCC y sobre todo los ligamentos, visualizados como estructuras lineales de baja señal ponderadas en T2. La línea punteada indica la transición convencional entre el bulbo raquídeo y la médula espinal. Abreviaciones: AAO, articulación atloido-odontoidea; $\mathrm{C} 1,1^{\text {a }}$ vértebra cervical; $C 2$, 2a vértebra cervical; $C 3$, 3a vértebra cervical; LLCA, ligamento longitudinal común anterior; LAAa, ligamento atloido-axoideo accesorio; LAp, ligamento apical; LT, ligamento trasverso; MOAA, membrana occipito-atloido-axoidea; MOAP, membrana occipito-atloidea posterior; MEc, médula espinal cervical; MT, membrana tectoria.

pequeño compartimiento sinovial interpuesto. El componente vertical del LC está formado por fibras delgadas que llegan arriba al clivus y abajo a la cara posterior de la AO y están aplicadas directamente a la MT situada por detrás. Este ligamento, sobre todo su componente transverso, juega un papel de gran jerarquía ya que asegura la posición del arco anterior de Cy su relación con la AO, evitando su desplazamiento posterior con la consiguiente compresión del eje neural.

Ligamentos alares: Son dos pequeñas estructuras que se extienden hacia arriba y afuera desde la AO, hacia el sector más interno de los $\mathrm{CO}^{6}{ }^{6} \mathrm{Si}$ bien su disposición oblicua es la más común, estudios de RM han demostrado que pueden ser verticales o incluso estar dispuestos en un plano casi horizontal. ${ }^{7}$ Su función es limitar la rotación axial e inclinación lateral durante los movimientos de flexoextensión de la UCC. Algunos autores señalan que frente a una rotura del LT, los LA son responsables de mantener cierto grado de estabilidad dada su disposición y desarrollo. ${ }^{4,6}$

Membrana tectoria: La MT es la extensión cefálica del LLP cuando éste llega al complejo C1-C2. Así está unida a la cara 


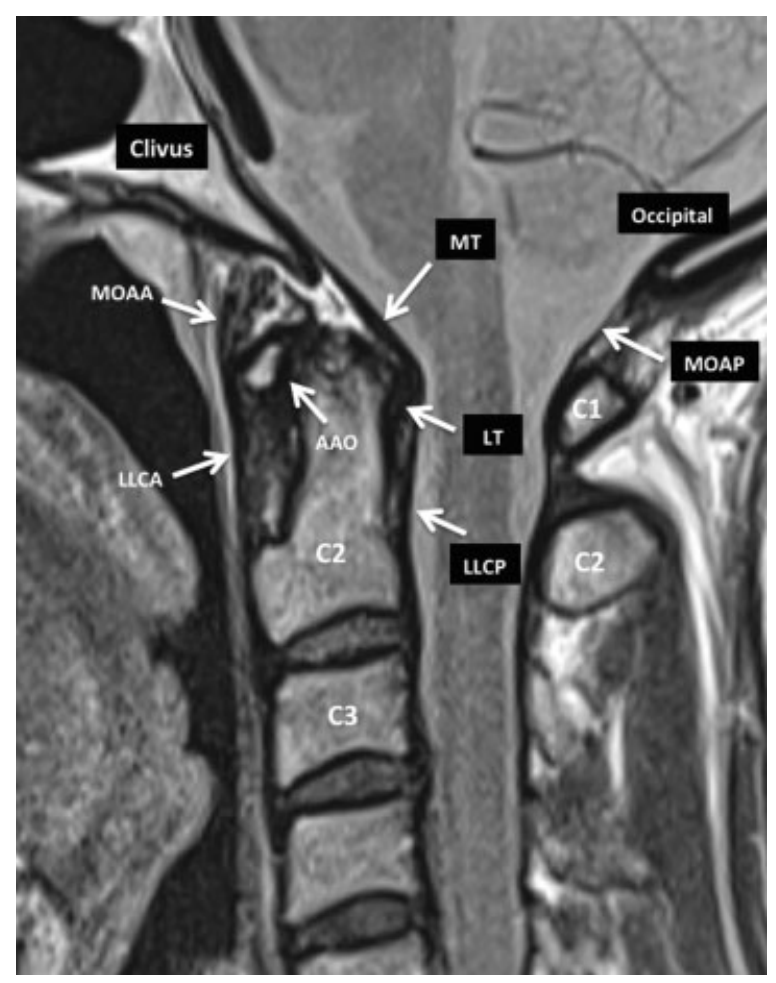

Fig. 9 RM, secuencia DP, plano sagital. Corte sagital de la UCC idéntico al de la figura 8 pero en secuencia DP que resalta las estructuras ligamentarias. Se destaca la identificación del LT situado entre la apófisis odontoidea de C2 y la MT. Abreviaciones: AAO, articulación atloido-odontoidea; $\mathrm{C} 1,1^{\text {a }}$ vértebra cervical; $C 2,2^{\text {a }}$ vértebra cervical; C3, 3a vértebra cervical; LLCA, ligamento longitudinal común anterior; LLCP, ligamento longitudinal común posterior; LT, ligamento trasverso; MOAA, membrana occipitoatloido-axoidea; MT, membrana tectoria; MOAP, membrana occipitoatloidea posterior.

posterior del cuerpo de C2 y se expande hacia arriba para unirse al borde anterior del agujero occipital donde se continúa con la duramadre craneana. ${ }^{8} \mathrm{Si}$ bien su rol funcional ha sido muy discutido, la mayoría de los autores sostienen que participa en la estabilización del raquis sobre todo durante los movimientos de flexión, y secundariamente en los movimientos de rotación. ${ }^{4,8}$

Ligamento longitudinal anterior: esa estructura, que recorre toda la cara anterior del raquis unida a los cuerpos vertebrales, arriba se inserta en el margen anterior del clivus del occipital. A ese nivel está formado por dos componentes: el ligamento occipito-atloideo (también llamado membrana occipitoatloide anterior), y el atloido-axoideo, menos desarrollado. Existe un ligamento o membrana occipito-atloidea posterior que une la protuberancia occipital al arco posterior de C1 pero juega un rol mecánico despreciable en la estabilidad de la región.

Ligamento apical: es una fina estructura fibrosa que se extiende desde la punta de la $\mathrm{AO}$ al basion del cráneo. Es denominado por algunos autores como ligamento suspensorio y está situado en el ángulo que se forma entre los LA. ${ }^{9}$ La mayoría de los autores están de acuerdo en que su contribución a la estabilidad también es despreciable, secundaria, y muy discutida. ${ }^{5,9}$ Uno de los puntos clave que sostienen este concepto es que estudios anatómicos encuentran este ligamento bien desarrollado en sólo un $80 \%$ de los especímenes cadavéricos. ${ }^{9}$

\section{Relaciones Anatómicas Fundamentales}

La UCC tiene relaciones anatómicas de gran jerarquía y que explican los déficits neurológicos o complicaciones que pueden producirse en el trauma que compromete esta región. El conducto raquídeo es muy amplio a este nivel y en su interior se da la transición entre el bulbo, parte baja del tronco encefálico, y la médula espinal. En ese sector del neuroeje, existen núcleos que regulan funciones vitales de gran importancia con el ciclo cardíaco y patrón respiratorio.

Al mismo tiempo transitan estructuras vasculares importantes como las arterias vertebrales (AV), que luego de ascender por el cuello a través de los agujeros transversos de las vértebras cervicales, realizan bucles, penetran la duramadre y ascienden a ambos lados del bulbo para ingresar al cráneo y dar origen al tronco basilar.

Esa relación anatómica, explica que en un porcentaje no despreciable de pacientes con trauma cervical, puedan producirse lesiones arteriales de entidad variable (sobre todo disecciones), con la consiguiente aparición de isquemia en el territorio correspondiente.

\section{Conceptos Biomecánicos Fundamentales}

La UCC es un complejo sistema articular que permite combinar solidez y extrema movilidad entre la cabeza y la columna vertebral. ${ }^{10-12}$ Así asegura el sostén cefálico, necesario para el mantenimiento en posición ideal de los órganos de los sentidos, y al mismo tiempo la amplitud de movimiento necesaria, en los 3 planos, para su correcta orientación. Su compleja anatomía surge como adaptación a la adquisición de la posición bípeda durante la evolución, de forma tal que el sentido de la visión puede tener a su alcance todo el espacio situado a nuestro alrededor. Como ya vimos, los movimientos se producen en los 3 planos del espacio: flexo-extensión, rotación axial y movimientos de inclinación lateral.

Previamente mencionamos que los movimientos de flexoextensión son los más amplios y se producen sobre todo en las AOA. ${ }^{10-12}$ Esos movimientos tienen una amplitud global de $20^{\circ}-$ $25^{\circ}$ y los de rotación axial e inclinación son mínimos. Los movimientos de rotación axial se producen en la AAO, acompañados por las AAA laterales y no superan los $8^{\circ}$. Durante la rotación, la AO actúa como pívot, manteniéndose casi inmóvil, siendo C1 la estructura que gira a su alrededor. ${ }^{10-12}$

Es importante entender cómo durante la flexo-extensión se produce un ascenso relativo de la AO, y se limita por su "impacto" en el borde anterior del foramen magno. En cambio, durante los movimientos de extensión, el LT y sobre todo la MT, estructuras dinámicas, evitan su desplazamiento posterior y protegen el neuroeje. ${ }^{6,8}$ Todos esos movimientos son acompañados y asegurados además por la movilidad y solidez del raquis cervical bajo (C3 a C7).

Los movimientos de inclinación lateral son mínimos o nulos en este complejo articular, y están limitados por las propias estructuras óseas de disposición lateral, sobre todo las AOA, así como también por los LA. 

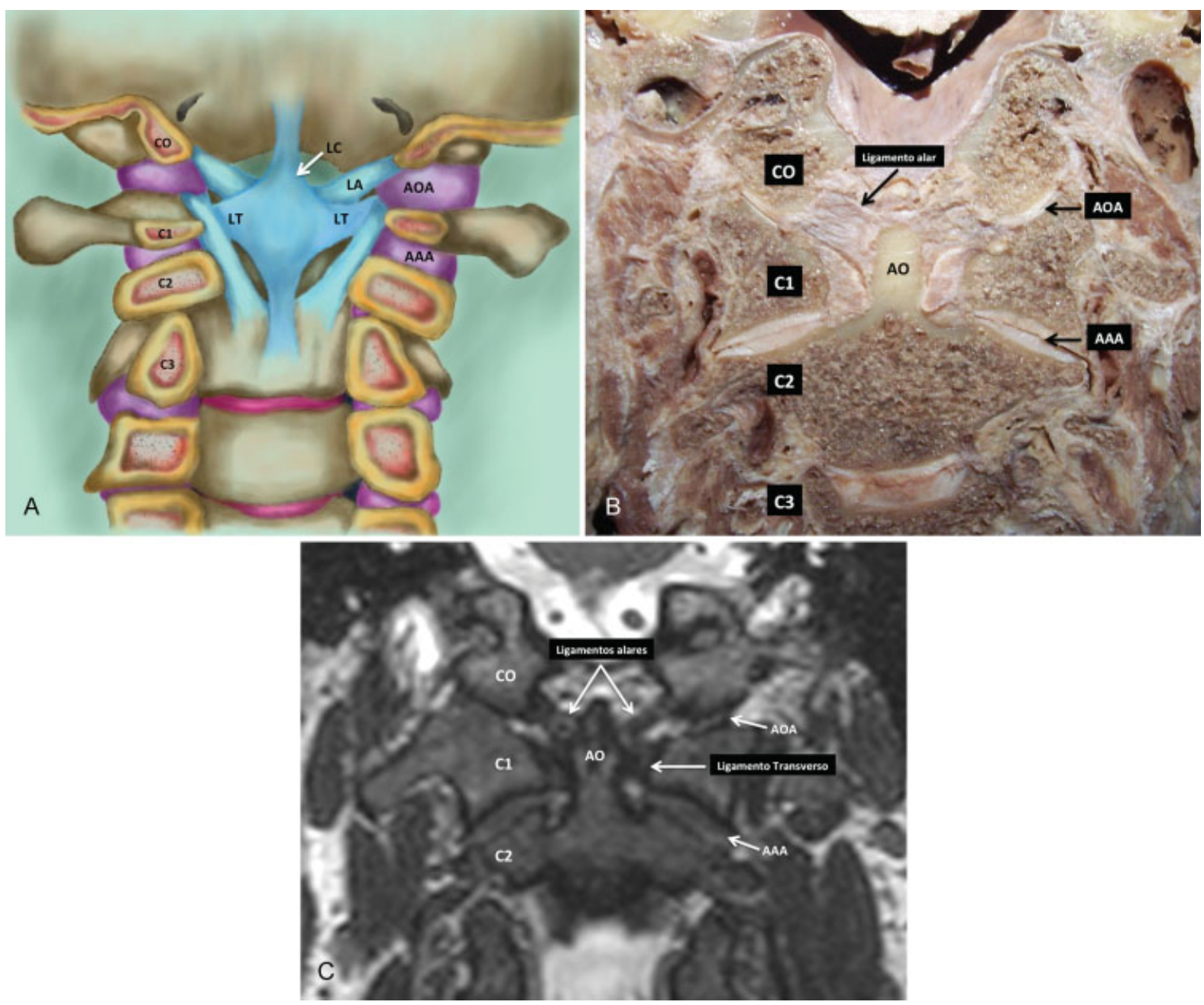

Fig. 10 Vista coronal de los ligamentos de la UCC. (a) Esquema en el que se representa un corte coronal, vista posterior, desde el conducto raquídeo, donde se muestra la disposición de los principales ligamentos de la UCC según se describe en el texto. (b) Corte cadavérico equivalente al esquema. (c) Secuencia cisternográfica 3D coronal Cube (GE). Abreviaciones: AAA, articulación atloido-axoidea; AOA, articulación occipito-

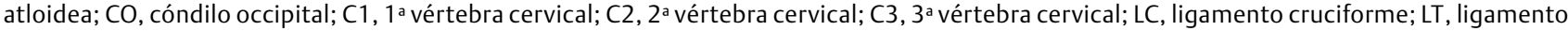
trasverso; LA, ligamento alar.

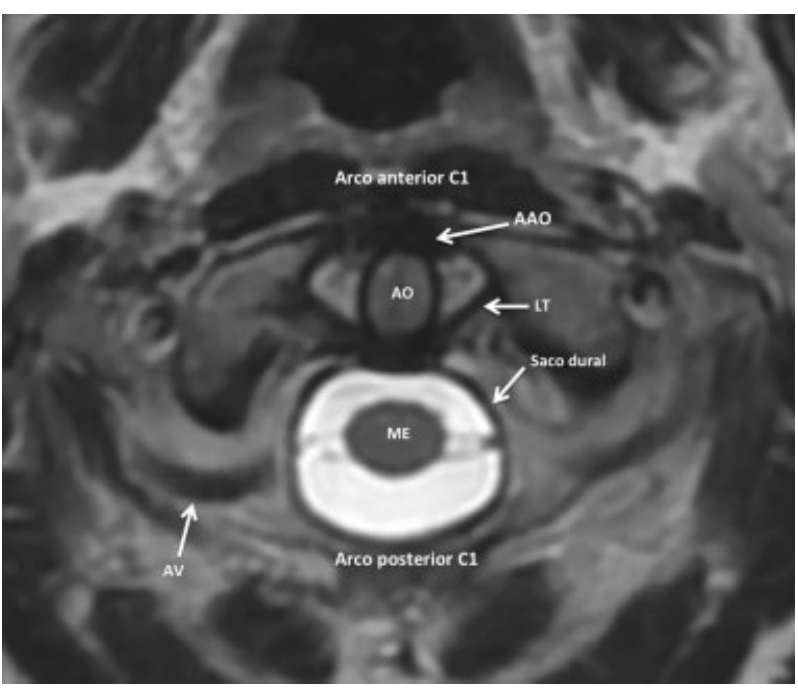

Fig. 11 RM, corte axial, secuencia CUBE 3D (GE, Healthcare) de la UCC. Se muestra en el corte axial la AAO con la disposición del LT. Abreviaciones: AO, apófisis odontoidea; AV, arteria vertebral; ME, médula espinal.

\section{Correlación Anátomo-radiológica}

En la actualidad, los avances tecnológicos tanto de tomografía computada multidetector (TCMD) como de resonancia magnética, permiten realizar un detallado análisis de cada uno de los componentes anatómicos de la UCC. Así, con TCMD se pueden obtener adquisiciones volumétricas con cortes finos, isotrópicas, para realizar luego reprocesamientos multi-planares y con técnica de volumen de superficie (VR). Con RM se pueden obtener imágenes en los 3 planos del espacio, con alta resolución anatómica, que permite ver con gran detalle sobre todo las estructuras ligamentarias de la UCC.

Se pueden utilizar secuencias potenciadas en $\mathrm{T} 2 \mathrm{y}$ densidad protónica (DP), sobre todo en el plano sagital y coronal, así como también secuencias de alta resolución, volumétricas, 3D e isotrópicas, con efecto cisternográfico (CUBE/CISS/SPACE/FIESTA). Esas secuencias de alta resolución se obtienen con cortes sub milimétricos y luego pueden reconstruirse en los 3 planos del espacio e incluso con oblicuidades específicas. 
En RM los ligamentos son complejos de identificar si no se logran imágenes paralelas a la disposición anatómica de las fibras, por lo que la planificación del estudio y la adquisición son fundamentales. ${ }^{13-15}$ También es necesario adquirir imágenes con espesores de cortes menores a $2 \mathrm{~mm}$, sin intervalo entre ellos (sin GAP) y con matriz de alta resolución para lograr una adecuada resolución espacial.

Los ligamentos se visualizan como estructuras de baja señal, o hipointensas, en secuencias potenciadas en T2/DP y cada uno de ellos se analizan en un plano del espacio diferente. $^{13-15}$ Por ejemplo el LT se visualiza mejor en el plano axial, el LA en el plano coronal mientras que el LAp y MT se observan en el plano sagital (- Fig. 11).

El conocimiento anatómico de la disposición de los diferentes ligamentos permite realizar reconstrucciones de las adquisiciones volumétricas de forma tal de analizar correctamente cada estructura. Debe recordarse que en situaciones de lesiones ligamentarias, si la RM es realizada en las primeras 48-72 horas del evento traumático, se produce un aumento de señal en ponderaciones $\mathrm{T} 2$ en el ligamento comprometido debido al aumento del contenido de agua en la estructura lesionada. ${ }^{14} \mathrm{~A}$ medida que evoluciona la lesión la intensidad de señal disminuye y el diagnóstico del compromiso ligamentario es más complejo.

\section{Conceptos Finales}

El trauma cervical es frecuente en la práctica clínica y las lesiones que involucran al raquis cervical alto o sub-occipital, pueden comprometer sectores del neuroeje cuya lesión determina una elevada morbi-mortalidad. La UCC está formada con un conjunto de articulaciones y ligamentos que aseguran la estabilidad del cráneo y al mismo tiempo una importante movilidad. Esta particularidad radica sobre todo en ligamentos cuya disposición 3D es compleja pero que pueden visualizarse con excelente detalle en estudios de RM.

Es fundamental un sólido conocimiento de la anatomía normal de la UCC, sobre todo de los diferentes ligamentos, de forma tal de poder analizar de forma correcta los estudios de imagen de uso corriente y así realizar un preciso diagnóstico en pacientes con trauma.

\section{Responsabilidades Éticas}

Protección de personas y animales. Los autores declaran que para esta investigación no se han realizado experimentos en seres humanos ni en animales.

Confidencialidad de los datos. Los autores declaran que han seguido los protocolos de su centro de trabajo sobre la publicación de datos de pacientes.
Derecho a la privacidad y consentimiento informado. Los autores declaran que en este artículo no aparecen datos de pacientes.

\section{Conflicto de Intereses}

Los autores declaran no tener ningún conflicto de interés, excepto el Dr. Sgarbi que declara como posible conflicto de interés, ser miembro del Comité Internacional de Revisores de la RAR.

\section{Bibliografía}

1 Offiah CE, Day E. The craniocervical junction: embryology, anatomy, biomechanics and imaging in blunt trauma. Insights Imaging 2017;8(01):29-47

2 Lopez AJ, Scheer JK, Leibl KE, Smith ZA, Dlouhy BJ, Dahdaleh NS. Anatomy and biomechanics of the craniovertebral junction. Neurosurg Focus 2015;38(04):E2

3 Martin MD, Bruner HJ, Maiman DJ. Anatomic and biomechanical considerations of the craniovertebral junction. Neurosurgery 2010;66(3, Suppl)2-6

4 Debernardi A, D'Aliberti G, Talamonti G, Villa F, Piparo M, Collice M. The craniovertebral junction area and the role of the ligaments and membranes. Neurosurgery 2011;68(02):291-301

5 Tubbs RS, Hallock JD, Radcliff V, et al. Ligaments of the craniocervical junction. J Neurosurg Spine 2011;14(06):697-709

6 Dvorak J, Schneider E, Saldinger P, Rahn B. Biomechanics of the craniocervical region: the alar and transverse ligaments. J Orthop Res 1988;6(03):452-461

7 Schmidt P, Mayer TE, Drescher R. Delineation of alar ligament morphology: comparison of magnetic resonance imaging at 1.5 and 3 Tesla. Orthopedics 2012;35(11):e1635-e1639

8 Tubbs RS, Kelly DR, Humphrey ER, et al. The tectorial membrane: anatomical, biomechanical, and histological analysis. Clin Anat 2007;20(04):382-386

9 Tubbs RS, Grabb P, Spooner A, Wilson W, Oakes WJ. The apical ligament: anatomy and functional significance. J Neurosurg 2000; 92(2, Suppl)197-200

10 White AA III, Panjabi MM. The clinical biomechanics of the occipitoatlantoaxial complex. Orthop Clin North Am 1978;9 (04):867-878

11 Menezes AH, Traynelis VC. Anatomy and biomechanics of normal craniovertebral junction (a) and biomechanics of stabilization (b). Childs Nerv Syst 2008;24(10):1091-1100

12 Steinmetz MP, Mroz TE, Benzel EC. Craniovertebral junction: biomechanical considerations. Neurosurgery 2010;66(3,Suppl)7-12

13 Schweitzer ME, Hodler J, Cervilla V, Resnick D. Craniovertebral junction: normal anatomy with MR correlation. AJR Am J Roentgenol 1992;158(05):1087-1090

14 Nidecker AE, Shen PY. Magnetic Resonance Imaging of the craniovertebral junction ligaments: normal anatomy and traumatic injury. J Neurol Surg B Skull Base 2016;77(05):388-395

15 Krakenes J, Kaale BR, Rorvik J, Gilhus NE. MRI assessment of normal ligamentous structures in the craniovertebral junction. Neuroradiology 2001;43(12):1089-1097 